\title{
The Thermodynamic Limit for an Imperfect Boson Gas
}

\author{
E. B. Davies \\ Mathematical Institute Oxford, England
}

Received April 7, 1972

\begin{abstract}
We give a rigorous treatment in the infinite volume limit of a model Hamiltonian representing an imperfect Boson gas. In particular we obtain the exact expression for the mean particle density in the infinite volume limit as a function of the chemical potential, and show that the density function has a singularity at the critical density for Bose-Einstein condensation. We prove that, unlike the ideal Boson gas, the imperfect Boson gas has the same behaviour in the infinite volume limit for the grand canonical ensemble as for the canonical ensemble, and is moreover stable under small perturbations. We finally exhibit the possibility of ordinary condensation and prove that a system in an intermediate situation between two pure phases consists of a simple mixture of the two phases involved.
\end{abstract}

\section{§ 1. Introduction and Notation}

We let $\Lambda \subseteq \mathscr{R}^{3}$ be an open region of unit volume with smooth boundary and for $L \geqq 1$ let

$$
\begin{gathered}
\Lambda_{L}=\{L x: x \in \Lambda\}, \\
\mathscr{H}^{L}=L^{2}\left(\Lambda_{L}\right)
\end{gathered}
$$

and let $\mathscr{F}^{L}$ be the symmetric Fock space constructed from $\mathscr{H}^{L}$. We let $S^{L}$ be a self-adjoint Hamiltonian on $\mathscr{H}^{L}$ with discrete spectrum and eigenvalues

$$
0=L^{-2} E_{0}<L^{-2} E_{1} \leqq L^{-2} E_{2} \leqq \cdots
$$

counted according to multiplicity. We suppose certain asymptotic conditions on the growth of the eigenvalues which are satisfied in the case $S^{L}=-\frac{1}{2} \Delta$, and denote by $H_{0}^{L}$ the free Hamiltonian on $\mathscr{F}^{L}$ constructed from $S^{L}$ in the usual manner.

The Hamiltonian that we consider in this paper is

$$
H_{\mu}^{L}=H_{0}^{L}-\mu N^{L}+L^{3} f\left(N^{L} / L^{3}\right)
$$

where $N^{L}$ is the number operator on $\mathscr{F}^{L}$ and $f$ is a continuously differentiable function on $[0, \infty)$ satisfying $f(0)=0$ and

$$
\lim _{x \rightarrow \infty} f^{\prime}(x)=+\infty \text {. }
$$


The density matrix for this Hamiltonian at the inverse temperature $\beta$ is

$$
\sigma_{\beta, \mu}^{L}=e^{-\beta H_{\mu}^{L}} / \operatorname{tr}\left[e^{-\beta H_{\mu}^{L}}\right]
$$

and the mean particle density in the infinite volume limit is

$$
\varrho(\beta, \mu)=\lim _{L \rightarrow \infty} L^{-3} \operatorname{tr}\left[\sigma_{\beta, \mu}^{L} N^{L}\right] .
$$

We show that this limit exists and give an explicit procedure for evaluating it.

We summarize the technique adopted. We define the function $\gamma_{L}$ by interpolation from the formula

where

$$
\operatorname{tr}\left[P_{n} \eta_{\beta, \mu_{0}}^{L}\right]=\exp \left[-\beta L^{3} \gamma_{L}\left(n / L^{3}\right)\right]
$$

$$
\eta_{\beta, \mu_{0}}^{L}=e^{-\beta\left(H_{0}^{L}-\mu_{0} N^{L}\right)} / \operatorname{tr}\left[e^{-\beta\left(H_{0}^{L}-\mu_{0} N^{L}\right)}\right] .
$$

Here $\mu_{0}$ is any fixed constant less than zero and $P_{n}$ is the projection of $\mathscr{F}^{L}$ onto its $n$-particle subspace. We prove that $\gamma_{L}$ is a convex function and that

$$
\lim _{L \rightarrow \infty} \gamma_{L}(x)=\gamma(x)
$$

where $\gamma$ is defined as follows. For each $\sigma \in \mathscr{R}$ we define the function $g_{\sigma}$ by

$$
g_{\sigma}(z)=\sum_{n=1}^{\infty} n^{-\sigma} z^{n}
$$

the series converging for $|z|<1$, and for $|z| \leqq 1$ if $\sigma>1$. We then define the critical density $\varrho_{c}$ by

$$
\varrho_{c}=(2 \pi \beta)^{-3 / 2} g_{3 / 2}(1) .
$$

The function $\gamma$ is defined for $0 \leqq x \leqq \varrho_{c}$ by

where

$$
\begin{aligned}
\gamma(x)=\left(\mu-\mu_{0}\right) x & -\beta^{-1}(2 \pi \beta)^{-3 / 2} g_{5 / 2}\left(e^{\beta \mu}\right) \\
& +\beta^{-1}(2 \pi \beta)^{-3 / 2} g_{5 / 2}\left(e^{\beta \mu_{0}}\right)
\end{aligned}
$$

$$
x=(2 \pi \beta)^{-3 / 2} g_{3 / 2}\left(e^{\beta \mu}\right)
$$

gives $\mu$ as a function of $\lambda$. We note that $\gamma^{\prime}\left(\varrho_{c}\right)=-\mu_{0}$ and define $\gamma$ for $\varrho_{c} \leqq x<\infty$ by

$$
\gamma(x)=\gamma\left(\varrho_{c}\right)-\mu_{0}\left(x-\varrho_{c}\right) .
$$

We comment in passing that results such as Eq. (1.9) have been obtained in general probabilistic situations $[1,2]$ but not, as far as we know, in a directly applicable form.

If we can define

$$
Q(n, \mu, \beta)=\operatorname{tr}\left[P_{n} e^{-\beta H_{\mu}^{L}}\right] / \operatorname{tr}\left[e^{-\beta H_{\mu}^{L}}\right]
$$


then it follows from Eq. (1.9) that as $L \rightarrow \infty$

$$
Q(n, \mu, \beta) \sim k_{L} \exp \left[-\beta L^{3}\left\{\gamma\left(n / L^{3}\right)+f\left(n / L^{3}\right)+\left(\mu_{0}-\mu\right) n / L^{3}\right\}\right]
$$

so that the particle number distribution is concentrated around the point or points where

$$
\gamma(x)+f(x)+\left(\mu_{0}-\mu\right) x
$$

takes its minimum value. We prove that $x=\varrho(\beta, \mu)$ is such a point and that the phase transitions occur for those values of $\mu$ where the function takes its minimum value at more than one point.

The main results of the paper are all contained in Section 4, the intermediate sections being devoted to technical preliminaries.

Before proceeding the author would like to thank J. T. Lewis, J. Pulè, J. F. C. Kingman and $\mathrm{M}$. Kac for many stimulating conversations and suggestions.

\section{§ 2. The Central Limit Theorem}

We apply here some methods developed systematically for the ideal Boson gas by Lewis and Pulè [3] from ideas of Kac [4]. Their basis is the fact that for the ideal Boson gas the different modes are independent and so explicit expressions for the mean and variance of the particle number distribution can be written down. Careful estimates then allow the calculation of the infinite volume limits of these expressions.

We first make it clear that our only assumptions are on the asymptotic behaviour of the eigenvalues $\left\{L^{-2} E_{n}\right\}_{n=0}^{\infty}$ of the one-particle Hamiltonian $S^{L}$. Specifically we assume that

$$
\lim _{x \rightarrow \infty}\left[x^{-3 / 2} \max \left\{m: E_{m} \leqq x\right\}\right]=\frac{\sqrt{2}}{3 \pi^{2}} .
$$

It is easy to show that this condition is satisfied if $S^{L}=-\frac{1}{2} \Delta$ on the cube $\Lambda_{L}=[0, L]^{3}$ with periodic boundary conditions. It is also satisfied for the same Hamiltonian if $\Lambda$ is a much more general region of unit volume with various boundary conditions $[3,5]$.

We define the distribution function $F_{L}$ by

so that

$$
F_{L}(x)=L^{-3}\left[\max \left\{m: L^{-2} E_{m} \leqq x\right\}-1\right]
$$

$$
\lim _{L \rightarrow \infty} F_{L}(x)=\frac{\sqrt{2}}{3 \pi^{2}} x^{3 / 2} .
$$

Easy calculations show that there is a constant $k$ independent of $L$ and $x$ such that

$$
F_{L}(x) \leqq k x^{3 / 2} .
$$

Our asymptotic estimates are based on the following lemma. 
Lemma 2.1. If $f$ is a continuously differentiable function on $[0, \infty)$ such that

$$
\lim _{m \rightarrow \infty} m^{2} f\left(L^{-2} E_{m}\right)=0
$$

then

$$
\begin{aligned}
& \text { Proof. } \sum_{m=0}^{\infty} f\left(L^{-2} E_{m}\right)=f(0)-L^{3} \int_{0}^{\infty} f^{\prime}(x) F_{L}(x) d x . \\
& \sum_{m=0}^{\infty} f\left(L^{-2} E_{m}\right)=\lim _{n \rightarrow \infty} \sum_{m=0}^{n} f\left(L^{-2} E_{m}\right) \\
& =\lim _{n \rightarrow \infty}\left\{f(0)-\int_{L^{-2} E_{1}}^{L^{-2} E_{2}} f^{\prime}(x) d x-2 \int_{L^{-2} E_{2}}^{L^{-2} E_{3}} f^{\prime}(x) d x\right. \\
& \left.-\cdots-(n-1) \int_{L^{-2} E_{n-1}}^{L^{-2} E_{n}} f^{\prime}(x) d x+n f\left(L^{-2} E_{n}\right)\right\} \\
& =\lim _{n \rightarrow \infty}\left\{f(0)-L^{3} \int_{0}^{L^{-2} E_{n}} f^{\prime}(x) F_{L}(x) d x+n f\left(L^{-2} E_{n}\right)\right\} \\
& =f(0)-L^{3} \int_{0}^{\infty} f^{\prime}(x) F_{L}(x) d x .
\end{aligned}
$$

We let $X_{n}^{L}$ be the random variable, concentrated on the non-negative integers, which describes the particle number distribution of the $n$th mode for the density matrix $\eta_{\beta, \mu_{0}}^{L}$, so that

$$
P\left(X_{n}^{L}=m\right)=\left\{1-e^{-\beta\left(L^{-2} E_{n}-\mu_{0}\right)}\right\} e^{-m \beta\left(L^{-2} E_{n}-\mu_{0}\right)} .
$$

The random variables $\left\{X_{n}^{L}\right\}_{n=0}^{\infty}$ are independent and if

then

$$
X^{L}=\sum_{n=0}^{\infty} X_{n}^{L}
$$

We let

and

$$
P\left(X^{L}=m\right)=\operatorname{tr}\left[P_{m} \eta_{\beta, \mu_{0}}^{L}\right] .
$$

Lemma 2.2. $\lim _{L \rightarrow \infty} m_{L}=(2 \pi \beta)^{-3 / 2} g_{3 / 2}\left(e^{\beta \mu_{0}}\right) \equiv \varrho_{0}$ (say) .

Proof. Since

putting

$$
m_{L}=L^{-3} \sum_{n=0}^{\infty} E\left(X_{n}^{L}\right)=L^{-3} \sum_{n=0}^{\infty} \frac{e^{-\beta\left(L^{-2} E_{n}-\mu_{0}\right)}}{1-e^{-\beta\left(L^{-2} E_{n}-\mu_{0}\right)}}
$$

$$
f(x)=L^{-3} \frac{e^{-\beta\left(x-\mu_{0}\right)}}{1-e^{-\beta\left(x-\mu_{0}\right)}}
$$


in Lemma (2.1) yields

$$
m_{L}=L^{-3} \frac{e^{\beta \mu_{0}}}{1-e^{\beta \mu_{0}}}+\int_{0}^{\infty} \frac{\beta e^{\beta x} e^{\beta \mu_{0}}}{\left(e^{\beta x}-e^{\beta \mu_{0}}\right)^{2}} F_{L}(x) d x .
$$

Using the Lebesgue dominated convergence theorem with Eqs. (2.3) and (2.4) implies

$$
\begin{aligned}
\lim _{L \rightarrow \infty} m_{L} & =\int_{0}^{\infty} \frac{\beta e^{\beta x} e^{\beta \mu_{0}}}{\left(e^{\beta x}-e^{\beta \mu_{0}}\right)^{2}} \frac{\sqrt{2} x^{3 / 2}}{3 \pi^{2}} d x \\
& =\int_{0}^{\infty} \frac{e^{\beta \mu_{0}}}{e^{\beta x}-e^{\beta \mu_{0}}} \frac{x^{1 / 2}}{\sqrt{2} \pi^{2}} d x \\
& =\sum_{n=1}^{\infty} e^{n \beta \mu_{0}} \frac{1}{\sqrt{2} \pi^{2}} \int_{0}^{\infty} e^{-n \beta x} x^{1 / 2} d x \\
& =(2 \pi \beta)^{-3 / 2} g_{3 / 2}\left(e^{\beta \mu_{0}}\right) .
\end{aligned}
$$

Lemma 2.3. $\quad \lim _{L \rightarrow \infty} s_{L}^{2}=(2 \pi \beta)^{-3 / 2} g_{1 / 2}\left(e^{\beta \mu_{0}}\right)$.

Proof. Since

putting

$$
s_{L}^{2}=L^{-3} \sum_{n=0}^{\infty} \operatorname{Var}\left(X_{n}^{L}\right)=L^{-3} \sum_{n=0}^{\infty} \frac{e^{-\beta\left(L^{-2} E_{n}-\mu_{0}\right)}}{\left\{1-e^{-\beta\left(L^{-2} E_{n}-\mu_{0}\right)}\right\}^{2}}
$$

in Lemma (2.1) yields

$$
f(x)=L^{-3} \frac{e^{-\beta\left(x-\mu_{0}\right)}}{\left(1-e^{-\beta\left(x-\mu_{0}\right)}\right)^{2}}
$$

$$
s_{L}^{2}=L^{-3} \frac{e^{\beta \mu_{0}}}{\left(1-e^{\beta \mu_{0}}\right)^{2}}-\int_{0}^{\infty} \frac{d}{d x}\left\{\frac{e^{-\beta\left(x-\mu_{0}\right)}}{\left(1-e^{-\beta\left(x-\mu_{0}\right)}\right)^{2}}\right\} F_{L}(x) d x .
$$

Using the Lebesgue dominated convergence theorem with Eqs. (2.3) and (2.4) implies

$$
\begin{aligned}
\lim _{L \rightarrow \infty} s_{L}^{2} & =-\int_{0}^{\infty} \frac{d}{d x}\left\{\frac{e^{-\beta\left(x-\mu_{0}\right)}}{\left(1-e^{-\beta\left(x-\mu_{0}\right)}\right)^{2}}\right\} \frac{\sqrt{2} x^{3 / 2}}{3 \pi^{2}} d x \\
& =\int_{0}^{\infty} \frac{e^{-\beta\left(x-\mu_{0}\right)}}{\left(1-e^{-\beta\left(x-\mu_{0}\right)}\right)^{2}} \frac{x^{1 / 2}}{\sqrt{2} \pi^{2}} d x \\
& =\sum_{n=1}^{\infty} n e^{n \beta \mu_{0}} \frac{1}{\sqrt{2} \pi^{2}} \int_{0}^{\infty} e^{-n \beta x} x^{1 / 2} d x \\
& =(2 \pi \beta)^{-3 / 2} g_{1 / 2}\left(e^{\beta \mu_{0}}\right) .
\end{aligned}
$$

Theorem 2.4. The random variables $X^{L}$ are asymptotically normally distributed, that is for all $x \leqq y$

$\lim _{L \rightarrow \infty} P\left(L^{3} m_{L}+L^{3 / 2} s_{L} x \leqq X^{L} \leqq L^{3} m_{L}+L^{3 / 2} s_{L} y\right)=\frac{1}{\sqrt{2 \pi}} \int_{x}^{y} e^{-t^{2} / 2} d t$. 
Proof. This is a consequence of Liapounov's condition for normal convergence of triangular arrays $[6,7]$. We have to prove that

$$
\lim _{L \rightarrow \infty} L^{-6} s_{L}^{-4} \sum_{n=0}^{\infty} E\left[\left\{X_{n}^{L}-E\left(X_{n}^{L}\right)\right\}^{4}\right]=0 .
$$

Now by direct calculations, for a random variable $Z$ such that

$$
P(Z=n)=(1-z) z^{n}
$$

we have

$$
E\left[\{Z-E(Z)\}^{4}\right]=\frac{z+7 z^{2}+z^{3}}{(1-z)^{4}} \leqq c \sum_{n=1}^{\infty} m^{3} z^{m} .
$$

Therefore

where

$$
\begin{aligned}
A^{L} & \equiv \sum_{n=0}^{\infty} E\left[\left\{X_{n}^{L}-E\left(X_{n}^{L}\right)\right\}^{4}\right] \\
& \leqq c \sum_{n=0}^{\infty} \sum_{m=1}^{\infty} m^{3} e^{-m \beta\left(L^{-2} E_{n}-\mu_{0}\right)} \\
& =c \sum_{m=1}^{\infty} m^{3} \varphi\left(L^{-2} m \beta\right) e^{m \beta \mu_{0}}
\end{aligned}
$$

$$
\begin{aligned}
\varphi\left(L^{-2} m \beta\right) & =\sum_{n=0}^{\infty} e^{-m \beta L^{-2} E_{n}} \\
& =1+L^{3} \int_{0}^{\infty} m \beta e^{-m \beta x} F_{L}(x) d x \\
& \leqq 1+L^{3} m \beta \int_{0}^{\infty} e^{-m \beta x} k x^{3 / 2} d x \\
& \leqq 1+a L^{3} m .
\end{aligned}
$$

Therefore

$$
A^{L} \leqq c \sum_{m=1}^{\infty} m^{3} e^{m \beta \mu_{0}}+a c L^{3} \sum_{m=1}^{\infty} m^{4} e^{m \beta \mu_{0}}
$$

and so by Lemma (2.3)

$$
\lim _{L \rightarrow \infty}\left(L^{-6} s_{L}^{-4} A^{L}\right)=0
$$

\section{§3. Asymptotic Behaviour of the Functions $\gamma_{L}$}

We investigate the properties of the function $\gamma$ defined by Eq. (1.7). We first note that since $X^{L}$ is a sum of independent geometric random variables, the sequence

$$
n \rightarrow e^{-L^{3} \gamma_{L}\left(n / L^{3}\right)}
$$


is a Polya frequency sequence [8] and hence $\gamma_{L}$ is a convex function of $n$. One may also verify this directly by induction. We extend $\gamma_{L}$ by interpolation to $[0, \infty)$ so that it is convex continuously differentiable and has the same minimum value as before. The following lemma yields the pressure function for the grand canonical ensemble [9].

Lemma 3.1. $\lim _{L \rightarrow \infty} \gamma_{L}(0)=\beta^{-1}(2 \pi \beta)^{-3 / 2} g_{5 / 2}\left(e^{\beta \mu_{0}}\right)$.

Proof. We have

$$
e^{-\beta L^{3} \gamma_{L}(0)}=P\left(X^{L}=0\right)=\prod_{n=0}^{\infty} P\left(X_{n}^{L}=0\right) .
$$

Therefore

$$
\gamma_{L}(0)=-\beta^{-1} L^{-3} \sum_{n=0}^{\infty} \log \left(1-e^{-\beta\left(L^{-2} E_{n}-\mu_{0}\right)}\right) .
$$

Putting

$$
f(x)=-\beta^{-1} L^{-3} \log \left(1-e^{-\beta\left(x-\mu_{0}\right)}\right)
$$

in Lemma (2.1) yields

$$
\gamma_{L}(0)=-\beta^{-1} L^{-3} \log \left(1-e^{\beta \mu_{0}}\right)+\beta^{-1} \int_{0}^{\infty} \frac{\beta e^{-\beta x} e^{\beta \mu_{0}}}{1-e^{-\beta\left(x-\mu_{0}\right)}} F_{L}(x) d x .
$$

Therefore by the Lebesgue dominated convergence theorem

$$
\begin{aligned}
\lim _{L \rightarrow \infty} \gamma_{L}(0) & =\int_{0}^{\infty} \frac{e^{-\beta x} e^{\beta \mu_{0}}}{1-e^{-\beta\left(x-\mu_{0}\right)}} \frac{\sqrt{2} x^{3 / 2}}{3 \pi^{2}} d x \\
& =\sum_{n=1}^{\infty} e^{n \beta \mu_{0}} \frac{\sqrt{2}}{3 \pi^{2}} \int_{0}^{\infty} e^{-n \beta x} x^{3 / 2} d x \\
& =\beta^{-1}(2 \pi \beta)^{-3 / 2} g_{5 / 2}\left(e^{\beta \mu_{0}}\right) .
\end{aligned}
$$

Lemma 3.2. Let $x_{L}$ be a point at which $\gamma_{L}$ attains its minimum value. Then

and

$$
\gamma_{L}^{\prime}\left(x_{L}\right)=0, \quad \lim _{L \rightarrow \infty} \gamma_{L}\left(x_{L}\right)=0
$$

$$
\lim _{L \rightarrow \infty} x_{L}=\varrho_{0} .
$$

Proof. We make use of Eq. (2.16) together with the fact that $\gamma_{L}$ is convex.

Putting $x=0$ and $y=1 / 5$ in Eq. (2.16) we find that for all large enough $L$

$$
\begin{aligned}
& \frac{1}{\sqrt{2 \pi}} \frac{1}{5} e^{-\frac{1}{50}} \leqq P\left(L^{3} m_{L} \leqq X^{L} \leqq L^{3} m_{L}+\frac{1}{5} L^{3 / 2} s_{L}\right) \\
& \quad \leqq \frac{1}{5} L^{3 / 2} s_{L} \exp \left[-\beta L^{3} \min \left\{\gamma_{L}\left(n / L^{3}\right): L^{3} m_{L} \leqq n \leqq L^{3} m_{L}+\frac{1}{5} L^{3 / 2} s_{L}\right\}\right] .
\end{aligned}
$$


Therefore

$$
\begin{gathered}
\beta L^{3} \min \left\{\gamma_{L}\left(n / L^{3}\right): m_{L} \leqq n / L^{3} \leqq m_{L}+\frac{1}{5} L^{-3 / 2} s_{L}\right\} \\
\leqq \frac{1}{50}+\log \left(\sqrt{2 \pi} L^{3 / 2} s_{L}\right) .
\end{gathered}
$$

Together with the obvious fact that $\gamma_{L}(x) \geqq 0$ for all $x$ this proves Eq. (3.5).

On the other hand putting $x=\frac{4}{5}$ and $y=1$ in Eq. (2.16) we find that for all large enough $L$

$\frac{1}{\sqrt{2 \pi}} \frac{1}{5} e^{-1 / 2} \geqq P\left(L^{3} m_{L}+\frac{4}{5} L^{3 / 2} s_{L} \leqq X^{L} \leqq L^{3} m_{L}+L^{3 / 2} s_{L}\right)$

$\geqq \frac{1}{5} L^{3 / 2} s_{L} \exp \left[-\beta L^{3} \max \left\{\gamma_{L}\left(n / L^{3}\right): L^{3} m_{L}+\frac{4}{5} L^{3 / 2} s_{L} \leqq n \leqq L^{3} m_{L}+L^{3 / 2} s_{L}\right\}\right]$.

Therefore

$$
\begin{gathered}
\beta L^{3} \max \left\{\gamma_{L}\left(n / L^{3}\right): m_{L}+\frac{4}{5} L^{-3 / 2} s_{L} \leqq n / L^{3} \leqq m_{L}+L^{-3 / 2} s_{L}\right\} \\
\geqq \frac{1}{2}+\log \left(\sqrt{2 \pi} L^{3 / 2} s_{L}\right) .
\end{gathered}
$$

By the convexity of $\gamma_{L}$ it follows from Eqs. (3.7) and (3.8) that if $x \geqq m_{L}$ $+L^{-3 / 2} s_{L}$ then

$$
\beta L^{3} \gamma_{L}(x) \geqq \frac{1}{2}+\log \left(\sqrt{2 \pi} L^{3 / 2} s_{L}\right) .
$$

The same result holds by similar arguments if $x \leqq m_{L}-L^{-3 / 2} s_{L}$. Therefore

$$
m_{L}-L^{-3 / 2} s_{L} \leqq x_{L} \leqq m_{L}+L^{-3 / 2} s_{L}
$$

from which Eq. (3.6) may be deduced.

Lemma 3.3. For all $0<x<\infty$ we have

Moreover

$$
\gamma_{L}^{\prime}(x) \leqq-\mu_{0} .
$$

$$
\lim _{x \rightarrow \infty} \gamma_{L}^{\prime}(x)=-\mu_{0} \text {. }
$$

Proof. We observe that for all $\mu<0, \eta_{\beta, \mu}^{L}$ is a well-defined density matrix and

$$
\begin{aligned}
\operatorname{tr}\left[P_{n} \eta_{\beta, \mu}^{L}\right] & =k_{L, \mu}^{-1} e^{-L^{3} \beta \gamma_{L}\left(n / L^{3}\right)} e^{\beta \mu n-\beta \mu_{0} n} \\
& =k_{L, \mu}^{-1} \exp \left[-L^{3} \beta\left\{\gamma_{L}\left(n / L^{3}\right)+\left(\mu_{0}-\mu\right) n / L^{3}\right\}\right]
\end{aligned}
$$

where

$$
k_{L, \mu}=\sum_{n=0}^{\infty} \exp \left[-L^{3} \beta\left\{\gamma_{L}\left(n / L^{3}\right)+\left(\mu_{0}-\mu\right) n / L^{3}\right\}\right]<\infty .
$$

It follows that

$$
\lim _{n \rightarrow \infty}\left\{\gamma_{L}\left(n / L^{3}\right)+\left(\mu_{0}-\mu\right) n / L^{3}\right\}=+\infty
$$

and so

$$
\lim _{x \rightarrow \infty}\left\{\gamma_{L}^{\prime}(x)+\left(\mu_{0}-\mu\right)\right\}>0
$$


Taking $\mu$ close to zero this implies

$$
\lim _{x \rightarrow \infty}\left\{\gamma_{L}^{\prime}(x)\right\} \geqq-\mu_{0} .
$$

On the other hand

$$
\begin{aligned}
& \lim _{\mu \uparrow 0} \operatorname{tr}\left[N^{L} \eta_{\beta, \mu}^{L}\right]=\lim _{\mu \uparrow 0} \sum_{n=0}^{\infty} E\left(X_{n, \mu}^{L}\right) \\
& \geqq \lim _{\mu \uparrow 0} E\left(X_{0, \mu}^{L}\right)=\lim _{\mu \uparrow 0} \frac{e^{\beta \mu}}{1-e^{\beta \mu}}=+\infty .
\end{aligned}
$$

Remembering that $\gamma_{L}^{\prime}$ is a monotonically increasing function, if Eq. (3.10) were false we should find that

$$
\lim _{\mu \uparrow 0} \operatorname{tr}\left[N^{L} \eta_{\beta, \mu}^{L}\right]=k_{L, 0}^{-1} \sum_{n=0}^{\infty} n e^{-L^{3} \beta\left\{\gamma_{L}\left(n / L^{3}\right)+\mu_{0} n / L^{3}\right\}}<\infty .
$$

The contradiction proves the validity of Eq. (3.10).

Lemma 3.4. For any $\mu>0$ if $u_{L}$ is a point at which

$$
\gamma_{L}(x)+\left(\mu_{0}-\mu\right) x
$$

attains its minimum value then

and

$$
\gamma_{L}^{\prime}\left(u_{L}\right)=\mu-\mu_{0}
$$

Proof. This is merely a repetition of the argument of Lemma (3.2) making use of Eq. (3.12). We note that this method does not allow us to find the limiting value of $\gamma_{L}\left(u_{L}\right)$ itself because we have no information on the constants $k_{L, \mu}$.

Lemma 3.5. $\gamma_{L}$ converges to $\gamma$ locally uniformly on the interval $\left\{x: 0<x<\varrho_{c}\right\}$.

Proof. We recall that $\gamma$ and $\varrho_{c}$ were defined in Eqs. (1.10) to (1.14) and observe that

$$
\gamma\left(\varrho_{0}\right)=0 .
$$

In view of Lemma (3.2) it is sufficient to prove that $\gamma_{L}^{\prime}$ converges locally uniformly to $\gamma^{\prime}$. For this purpose we note that explicit calculations show that

$$
\gamma^{\prime}(x)=\mu(x)-\mu_{0}
$$

where $\mu(x)$ is the inverse function of

$$
x=(2 \pi \beta)^{-3 / 2} g_{3 / 2}\left(e^{\beta \mu}\right)
$$

if $0 \leqq x \leqq \varrho_{c}$ and

$$
\mu(x)=0
$$

if $\varrho_{c} \leqq x<\infty$. 
One sees that $\mu(x)$ is continuous, monotonically increasing and that

$$
\lim _{x \downarrow 0} \mu(x)=-\infty, \quad \lim _{x \uparrow \varrho_{c}} \mu(x)=0 .
$$

Moreover $\gamma_{L}^{\prime}$ is monotonically increasing for all $L$ and we have shown in Lemma (3.4) that for all $x$ such that $0<x<\varrho_{c}$ there is a sequence $u_{L}$ such that

$$
\lim _{L \rightarrow \infty} u_{L}=x, \quad \lim _{L \rightarrow \infty} \gamma_{L}^{\prime}\left(u_{L}\right)=\gamma^{\prime}(x) .
$$

Let $0<x<\varrho_{c}$ and let $\varepsilon>0$. Choose $\delta>0$ small enough so that

$$
\left|\gamma^{\prime}(x \pm \delta)-\gamma^{\prime}(x)\right|<\varepsilon / 3
$$

and then choose $L_{0}$ large enough so that for all $L \geqq L_{0}$

and

$$
\left|\gamma_{L}^{\prime}\left(u_{L}\right)-\gamma^{\prime}(x+\delta)\right|<\varepsilon / 3
$$

$$
\left|\gamma_{L}^{\prime}\left(v_{L}\right)-\gamma^{\prime}(x-\delta)\right|<\varepsilon / 3
$$

where $\lim _{L \rightarrow \infty} u_{L}=x+\delta$ and $\lim _{L \rightarrow \infty} v_{L}=x-\delta$. Choose $L_{1} \geqq L_{0}$ large enough so that for all $L \geqq L_{1}$

$$
\left|u_{L}-(x+\delta)\right|<\delta / 2, \quad\left|v_{L}-(x-\delta)\right|<\delta / 2 .
$$

Then if $L \geqq L_{1}$ and $|y-x|<\delta / 2$

and

$$
\gamma_{L}^{\prime}(y) \leqq \gamma_{L}^{\prime}\left(u_{L}\right)<\gamma^{\prime}(x+\delta)+\varepsilon / 3<\gamma^{\prime}(y)+\varepsilon
$$

$$
\gamma_{L}^{\prime}(y) \geqq \gamma_{L}^{\prime}\left(v_{L}\right)>\gamma^{\prime}(x-\delta)-\varepsilon / 3>\gamma^{\prime}(y)-\varepsilon .
$$

This is enough to prove locally uniform convergence by standard arguments.

Lemma 3.6. $\gamma_{L}$ converges uniformly to $\gamma$ on the interval $\left\{x: 0 \leqq x \leqq \varrho_{0}\right\}$.

Proof. Some care is needed since $\gamma^{\prime}(0)=-\infty$. We use the previous lemma, the convexity of $\gamma_{L}$ and $\gamma$, and Lemma (3.1), which states that

$$
\lim _{L \rightarrow \infty} \gamma_{L}(0)=\gamma(0)
$$

We take $\varepsilon>0$ and choose $\delta>0$ small enough so that for all $0 \leqq x \leqq \delta$

$$
\gamma(x)>\gamma(0)-\varepsilon / 3 \text {. }
$$

Then we choose $L_{0}$ large enough so that for all $L \geqq L_{0}$

$$
\begin{aligned}
& \left|\gamma_{L}(0)-\gamma(0)\right|<\varepsilon / 3, \\
& \left|\gamma_{L}(\delta)-\gamma(\delta)\right|<\varepsilon / 3 .
\end{aligned}
$$


Then for all $0 \leqq x \leqq \delta$

$$
\begin{aligned}
\gamma_{L}(x) & \leqq \frac{x}{\delta} \gamma_{L}(\delta)+\frac{\delta-x}{\delta} \gamma_{L}(0) \\
& <\frac{x}{\delta} \gamma(\delta)+\frac{\delta-x}{\delta} \gamma(0)+2 \varepsilon / 3 \\
& \leqq \gamma(0)+2 \varepsilon / 3<\gamma(x)+\varepsilon .
\end{aligned}
$$

Together with Lemma (3.5) this implies that there exists $L_{1}$ such that for all $L \geqq L_{1}$ and all $0 \leqq x \leqq \varrho_{0}$

$$
\gamma_{L}(x)<\gamma(x)+\varepsilon .
$$

On the other hand since $\gamma_{L}^{\prime}$ is monotonically increasing and $\lim _{L \rightarrow \infty} \gamma_{L}^{\prime}\left(\varrho_{0}\right)=\gamma^{\prime}\left(\varrho_{0}\right)=0$, there exists a constant $L_{0}$ such that for all $0<x<\varrho_{0}$ and all $L \geqq L_{0} \quad \gamma_{L}^{\prime}(x)<1$.

Given $\varepsilon>0$ choose $\delta$ such that $0<\delta<\varepsilon / 4$ and

$$
|\gamma(x)-\gamma(0)|<\varepsilon / 4
$$

for all $0 \leqq x \leqq \delta$. Then choose $L_{0}$ large enough so that for all $L \geqq L_{0}$

$$
\left|\gamma_{L}(\delta)-\gamma(\delta)\right|<\varepsilon / 4
$$

Then if $0 \leqq x \leqq \delta$

$$
\begin{aligned}
\gamma_{L}(x) & \geqq \gamma_{L}(\delta)-(\delta-x) \\
& >\gamma_{L}(\delta)-\varepsilon / 4 \\
& >\gamma(\delta)-2 \varepsilon / 4 \\
& >\gamma(x)-\varepsilon .
\end{aligned}
$$

Together with Lemma (3.5) this implies that there exists $L_{1}$ such that for all $L \geqq L_{1}$ and all $0 \leqq x \leqq \varrho_{0}$

$$
\gamma_{L}(x)>\gamma(x)-\varepsilon
$$

and so completes the proof of the lemma.

Lemma 3.7. $\gamma_{L}$ converges uniformly to $\gamma$ on the interval

and

$$
\left\{x: \varrho_{0} \leqq x \leqq \varrho_{c}\right\}
$$

$$
\lim _{L \rightarrow \infty} \gamma_{L}^{\prime}\left(\varrho_{c}\right)=\gamma^{\prime}\left(\varrho_{c}\right)=-\mu_{0} .
$$

Proof. Since $\gamma_{L}^{\prime}$ are monotonically increasing and

$$
\lim _{L \rightarrow \infty} \gamma_{L}^{\prime}\left(\varrho_{0}\right)=\gamma^{\prime}\left(\varrho_{0}\right)=0
$$


there is a constant $L_{0}$ such that for all $L \geqq L_{0}$ and all $x$ such that $\varrho_{0} \leqq x<\infty$

$$
\gamma_{L}^{\prime}(x) \geqq-1 \text {. }
$$

On the other hand by Lemma (3.3)

$$
\gamma_{L}^{\prime}(x) \leqq-\mu_{0}
$$

for all $0 \leqq x<\infty$. Therefore the $\gamma_{L}$ are equicontinuous on $\left\{x: \varrho_{0} \leqq x \leqq \varrho_{c}\right\}$ and the convergence is uniform.

To prove Eq. (3.23) we observe that $\gamma^{\prime}\left(\varrho_{c}\right)=-\mu_{0}$ by direct calculations. Given $\varepsilon>0$ we choose $\delta>0$ small enough so that

$$
\left|\gamma^{\prime}(x)-\gamma^{\prime}\left(\varrho_{c}\right)\right|<\varepsilon / 3
$$

for all $\varrho_{c}-\delta \leqq x \leqq \varrho_{c}$. We then choose $L_{0}$ large enough so that

and

$$
\left|\gamma_{L}\left(\varrho_{c}-\delta\right)-\gamma\left(\varrho_{c}-\delta\right)\right|<\varepsilon \delta / 3
$$

for all $L \geqq L_{0}$.

$$
\left|\gamma_{L}\left(\varrho_{c}\right)-\gamma\left(\varrho_{c}\right)\right|<\varepsilon \delta / 3
$$

Then for some $v_{L}$ such that $\varrho_{0}-\delta<v_{L}<\varrho_{0}$

$$
\begin{aligned}
\gamma_{L}^{\prime}\left(v_{L}\right)= & \delta^{-1}\left\{\gamma_{L}\left(\varrho_{c}\right)-\gamma_{L}\left(\varrho_{c}-\delta\right)\right\} \\
& >\delta^{-1}\left\{\gamma\left(\varrho_{c}\right)-\gamma\left(\varrho_{c}-\delta\right)\right\}-2 \varepsilon / 3 \\
& >\gamma^{\prime}\left(\varrho_{c}\right)-\varepsilon .
\end{aligned}
$$

Combining the fact that $\gamma_{L}^{\prime}$ are monotonically increasing with Eq. (3.24) we obtain

$$
\gamma^{\prime}\left(\varrho_{c}\right) \geqq \gamma_{L}^{\prime}\left(\varrho_{c}\right) \geqq \gamma_{L}^{\prime}\left(v_{L}\right)>\gamma^{\prime}\left(\varrho_{c}\right)-\varepsilon
$$

which proves the lemma.

Lemma 3.8. For any $\varepsilon>0$ there exists a constant $L_{0}$ such that for any $L \geqq L_{0}$ and any $x \geqq \varrho_{c}$

$$
\begin{aligned}
& \gamma\left(\varrho_{c}\right)-\varepsilon+\left(\gamma^{\prime}\left(\varrho_{c}\right)-\varepsilon\right)\left(x-\varrho_{c}\right)<\gamma_{L}(x) \\
&<\gamma\left(\varrho_{c}\right)+\varepsilon+\gamma^{\prime}\left(\varrho_{c}\right)\left(x-\varrho_{c}\right) .
\end{aligned}
$$

In particular ${ }_{i_{L}}$ converges locally uniformly to $\gamma$ on the set $\left\{x: \varrho_{c} \leqq x<\infty\right\}$.

Proof. This is a simple consequence of the facts that

$$
\lim _{L \rightarrow \infty} \gamma_{L}\left(\varrho_{c}\right)=\gamma\left(\varrho_{c}\right)
$$

and for all $x>\varrho_{c}$ and all large enough $L$

$$
\gamma^{\prime}\left(\varrho_{c}\right) \geqq \gamma_{L}^{\prime}(x) \geqq \gamma_{L}^{\prime}\left(\varrho_{c}\right)>\gamma^{\prime}\left(\varrho_{c}\right)-\varepsilon .
$$


To summarize we have proved that $\gamma_{L}$ converges locally uniformly to $\gamma$ on the set

$$
\{x: 0 \leqq x<\infty\}
$$

and that for large $x$ the convergence is controlled by the estimates of Lemma (3.8).

\section{§ 4. The Thermodynamic Limit}

We recall from Section 1 that

$$
Q(n, \mu, \beta)=k_{L} \exp \left[-\beta L^{3}\left\{\gamma_{L}\left(n / L^{3}\right)+f\left(n / L^{3}\right)+\left(\mu_{0}-\mu\right) n / L^{3}\right\}\right] .
$$

An important role is clearly played by the points at which the function

$$
\gamma(x)+f(x)+\left(\mu_{0}-\mu\right) x
$$

takes its minimum value. We clarify this point before proceeding with the main results of the paper.

Theorem 4.1. Let $\varrho(\beta, \mu)$ be the smallest value of $x$ at which the function

$$
\gamma(x)+f(x)+\left(\mu_{0}-\mu\right) x
$$

takes its minimum value. Then for all real $\mu$

$$
0<\varrho(\beta, \mu)<\infty
$$

and $\varrho$ is a monotonically increasing function of $\mu$ such that

$$
\lim _{\mu \rightarrow-\infty} \varrho(\beta, \mu)=0, \quad \lim _{\mu \rightarrow \infty} \varrho(\beta, \mu)=\infty .
$$

Moreover $\varrho$ has a discontinuity at the point $\mu$ if and only if the function takes its minimum value at more than one value of $x$. There are only a countable number of such discontinuity points.

Proof. Eq. (4.4) is a consequence of

$$
\gamma^{\prime}(0)=-\infty, \quad \lim _{x \rightarrow \infty} f^{\prime}(x)=\infty .
$$

The remaining statements of the theorem follow by elementary analysis which we leave to the reader.

Notes. (i) Since we have explicitly written down the function $\gamma$ in Eq. (1.10) to (1.14), the function $\varrho$ can be effectively computed as soon as $f$ is given.

(ii) Differentiability properties of $\varrho$ in its interval of continuity depend on corresponding conditions for $f$, since $\gamma$ is an analytic function. 
(iii) The function $\varrho$ has a singularity where

$$
\varrho(\beta, \mu)=\varrho_{c}
$$

since $\gamma^{\prime \prime \prime}(x)$ is discontinuous at $x=\varrho_{c}$.

We are now able to prove the first main result of the paper.

Theorem 4.2. Let $\mu$ be a point of continuity of $\varrho(\beta, \mu)$. Then

$$
\lim _{L \rightarrow \infty} \frac{1}{L^{3}} \operatorname{tr}\left[N^{L} \sigma_{\beta, \mu}^{L}\right]=\varrho(\beta, \mu)
$$

and the particle number distribution concentrates entirely at the density $\varrho(\beta, \mu)$ in the infinite volume limit.

Proof. We let $A$ be the minimum value of the function of Formula (4.2). If $\varepsilon>0$ there exists a constant $\delta>0$ such that

if

$$
\gamma(x)+f(x)+\left(\mu_{0}-\mu\right) x \geqq A+\delta
$$

$$
|x-\varrho(\beta, \mu)| \geqq \varepsilon
$$

while there is a constant $\varepsilon_{1}$ such that $0<\varepsilon_{1}<\varepsilon$ and

if

$$
A \leqq \gamma(x)+f(x)+\left(\mu_{0}-\mu\right) x \leqq A+\delta / 2
$$

$$
|x-\varrho(\beta, \mu)|<\varepsilon_{1} \text {. }
$$

By Eqs. (1.14) and (4.5) there are constants $a>\varrho(\beta, \mu)+\varepsilon$ and $B>0$ such that if $x \geqq a$

$$
\gamma(x)+f(x)+\left(\mu_{0}-\mu\right) x \geqq A+\delta+B(x-a) .
$$

By Lemmas (3.6), (3.7) and (3.8) we can also suppose these inequalities are satisfied by $\gamma_{L}$ for all large enough $L$.

Let $Y^{L}$ be the random variable concentrated on the points $\left\{n / L^{3}: n=0,1,2, \ldots\right\}$ and given by

$$
P\left(Y^{L}=n / L^{3}\right)=\operatorname{tr}\left[P_{n} \sigma_{\beta, \mu}^{L}\right] \equiv Q(n, \mu, \beta) .
$$

Then for large enough $L$

$$
\begin{aligned}
a_{1, L} & \equiv P\left(0 \leqq Y^{L}<\varrho(\beta, \mu)-\varepsilon\right) \\
& \leqq L^{3}\{\varrho(\beta, \mu)-\varepsilon\} k_{L} \exp \left[-\beta L^{3}(A+\delta)\right], \\
b_{L} & \equiv P\left(\varrho(\beta, \mu)-\varepsilon \leqq Y^{L}<\varrho(\beta, \mu)+\varepsilon\right) \\
& \geqq L^{3} 2 \varepsilon_{1} k_{L} \exp \left[-\beta L^{3}(A+\delta / 2)\right], \\
a_{2, L} & \equiv P\left(\varrho(\beta, \mu)+\varepsilon \leqq Y^{L}<a\right) \\
& \leqq L^{3}\{a-\varrho(\beta, \mu)-\varepsilon\} k_{L} \exp \left[-\beta L^{3}(A+\delta)\right], \\
c_{m, L} & \equiv P\left(a+m \leqq Y^{L}<a+m+1\right) \\
& \leqq L^{3} k_{L} \exp \left[-\beta L^{3}(A+\delta+B m)\right] .
\end{aligned}
$$


These lead to the equations

$$
\begin{aligned}
& a_{1, L} b_{L}^{-1} \leqq \frac{\varrho(\beta, \mu)-\varepsilon}{2 \varepsilon_{1}} \exp \left[-\beta L^{3} \delta / 2\right], \\
& a_{2, L} b_{L}^{-1} \leqq \frac{a-\varrho(\beta, \mu)-\varepsilon}{2 \varepsilon_{1}} \exp \left[-\beta L^{3} \delta / 2\right], \\
& c_{m, L} b_{L}^{-1} \leqq \frac{1}{2 \varepsilon_{1}} \exp \left[-\beta L^{3} \delta / 2-\beta L^{3} B m\right],
\end{aligned}
$$

which do not involve the unknown constants $k_{L}$. Since $Y^{L}$ is a random variable

$$
a_{1, L}+b_{L}+a_{2, L}+\sum_{m=0}^{\infty} c_{m, L}=1 .
$$

It is easy to prove from Eqs. (4.16) to (4.19) that

$$
\begin{gathered}
\lim _{L \rightarrow \infty} b_{L}=1, \\
\limsup _{L \rightarrow \infty} E\left(Y^{L}\right) \leqq \varrho(\beta, \mu)+\varepsilon, \\
\liminf _{L \rightarrow \infty} E\left(Y^{L}\right) \geqq \varrho(\beta, \mu)-\varepsilon .
\end{gathered}
$$

Since $\varepsilon>0$ is arbitrary the theorem is proved.

We have shown that at points of continuity of $\varrho$, the particle density is entirely concentrated around one point, so that the canonical ensemble has the same behaviour as the grand canonical ensemble, even above the critical density for Bose-Einstein condensation. This is in sharp distinction to the situation for the ideal Boson gas $[3,10]$. Our calculations in fact prove that the limiting behaviour of the ideal Boson gas is not stable under small perturbations by functions of the number operator, while it is clear from the explicit form of our solution that the imperfect Boson gas is stable under such perturbations.

We finally consider the phase transitions themselves. To do this we have to go to the infinite volume limit keeping the mean density fixed instead of the chemical potential. We therefore suppose that for some value of $\mu$, say $\mu=\mu_{1}$, the function

$$
\gamma(x)+f(x)+\left(\mu_{0}-\mu\right) x
$$

takes its minimum value at exactly two points $x=\varrho_{1}$ and $x=\varrho_{2}$. (Other types of behaviour, although theoretically possible, are not stable under small perturbations of $f$.) We then consider a density $\varrho$ such that $\varrho_{1}<\varrho<\varrho_{2}$ and define the chemical potential $\mu(L)$ for the volume $L^{3}$ by

$$
L^{-3} \operatorname{tr}\left[N^{L} \sigma_{\beta, \mu(L)}^{L}\right]=\varrho .
$$


Theorem 4.3. In the limit as $L \rightarrow \infty$ the particle number distribution of $\sigma_{\beta, \mu(L)}^{L}$ is entirely concentrated at the points $\varrho_{1}$ and $\varrho_{2}$, in such a proportion that the mean density is $\varrho$.

Proof. We first observe that

$$
\begin{aligned}
\varrho_{L}(\beta, \mu) & =L^{-3} \operatorname{tr}\left[N^{L} \sigma_{\beta, \mu}^{L}\right] \\
& =\left\{\sum_{n=0}^{\infty} n e^{n \beta \mu} Q(n, 0, \beta)\right\} /\left\{\sum_{n=0}^{\infty} e^{n \beta \mu} Q(n, 0, \beta)\right\}
\end{aligned}
$$

is a monotonically increasing continuous function of $\mu$ which we have shown converges to $\varrho(\beta, \mu)$ at all points of continuity of the latter.

Since

$$
\varrho_{L}(\beta, \mu(L))=\varrho=\varrho\left(\beta, \mu_{1}\right)
$$

it follows by simple arguments that

$$
\lim _{L \rightarrow \infty} \mu(L)=\mu_{1}
$$

We carry out calculations similar to those of Theorem (4.2) with the following variations.

Instead of Eq. (4.9) we let $\varepsilon_{1}$ be a constant such that $0<\varepsilon_{1}<\varepsilon$ and

if

$$
A \leqq \gamma(x)+f(x)+\left(\mu_{0}-\mu\right) x \leqq A+\delta / 2
$$

$$
\left|x-\varrho_{i}\right|<\varepsilon_{1}
$$

for $i=1$ or $i=2$. Putting

$$
\begin{aligned}
& a_{1, L}=P\left(0 \leqq Y^{L}<\varrho_{1}-\varepsilon\right), \\
& b_{1, L}=P\left(\varrho_{1}-\varepsilon \leqq Y^{L}<\varrho_{1}+\varepsilon\right), \\
& a_{2, L}=P\left(\varrho_{1}+\varepsilon \leqq Y^{L}<\varrho_{2}-\varepsilon\right), \\
& b_{2, L}=P\left(\varrho_{2}-\varepsilon \leqq Y^{L}<\varrho_{2}+\varepsilon\right), \\
& a_{3, L}=P\left(\varrho_{2}+\varepsilon \leqq Y^{L}<a\right), \\
& c_{m, L}=P\left(a+m \leqq Y^{L}<a+m+1\right),
\end{aligned}
$$

we find as before estimates of the form

$$
\begin{aligned}
& a_{i, L} /\left(b_{1, L}+b_{2, L}\right) \leqq d_{i} \exp \left[-\beta L^{3} \delta / 2\right], \\
& c_{m, L} /\left(b_{1, L}+b_{2, L}\right) \leqq e \exp \left[-\beta L^{3} \delta / 2-\beta L^{3} B m\right],
\end{aligned}
$$

from which it follows that

$$
\lim _{L \rightarrow \infty}\left(b_{1, L}+b_{2, L}\right)=1 .
$$


Now if $u=\limsup _{L \rightarrow \infty} b_{1, L}$ and $v=\liminf _{L \rightarrow \infty} b_{1, L}$ then since $E\left(Y^{L}\right)=\varrho$ for all $L$

and

$$
\varrho \leqq\left(\varrho_{1}+\varepsilon\right) v+\left(\varrho_{2}+\varepsilon\right)(1-v)
$$

Therefore

$$
\varrho \geqq\left(\varrho_{1}-\varepsilon\right) u+\left(\varrho_{2}-\varepsilon\right)(1-u) \text {. }
$$

$$
\frac{\varrho_{2}-\varrho-\varepsilon}{\varrho_{2}-\varrho_{1}} \leqq v \leqq u \leqq \frac{\varrho_{2}-\varrho+\varepsilon}{\varrho_{2}-\varrho_{1}} \text {. }
$$

Since $\varepsilon>0$ is arbitrary one may conclude that

$$
\lim _{L \rightarrow \infty} b_{1, L}=\frac{\varrho_{2}-\varrho}{\varrho_{2}-\varrho_{1}}, \quad \lim _{L \rightarrow \infty} b_{2, L}=\frac{\varrho-\varrho_{1}}{\varrho_{2}-\varrho_{1}}
$$

and the theorem is proved.

We finally discuss the case where $f$ is convex, which is expecially simple. We point out that the particular case

$$
f(x)=A x^{2}
$$

has already been studied in some detail [11].

Theorem 4.4. If $f$ is a strictly convex function then $\varrho(\beta, \mu)$ is a continuous function of $\mu$. The only phase transition occurs at $\varrho=\varrho_{c}$ and corresponds to the commencement of Bose-Einstein condensation. Below the critical density $\varrho=\varrho_{c}$ one has $\varrho(\beta, \mu)=x$ if and only if

$$
f^{\prime}(x)+\mu(x)=\mu
$$

while above the critical density the corresponding condition is

$$
f^{\prime}(x)=\mu .
$$

Proof. If $f$ is strictly convex then the function of Formula (4.2) is strictly convex and takes its minimum at exactly one point, the point where its derivative vanishes. The remainder of the theorem is then a restatement of known results.

The determination of the mean potential function $f$ for a given system is a highly non-trivial matter. In principle it should be possible to find $f$ given a pair interaction potential $\Phi$, but this is an unsolved problem. Supposing that $\Phi$ is weakly attractive at long range and strongly repulsive at short range, and that $f(x)$ is the sum of the potentials due to the individual particles in an "average" configuration of density $x$, one would expect that $f(x)$ would be small and negative at low density, but large and positive at high density.

As a simple typical mean potential of this type one may consider

$$
f(x)=-a^{2} x^{2}+b^{2} x^{4} .
$$


It is straightforward to carry out the calculations described in this section for such a function. One finds that at high temperature (small $\beta$ ) the function $(f+\gamma)$ is convex, so there is no phase transition. However, below a certain critical temperature $(f+\gamma)$ is not convex and a phase transition does occur. These results are in qualitative agreement with physical expectations.

\section{References}

1. Bahadue, R.R., Ranga Rao, R.: On deviations in the sample mean. Ann. Math. Stat. 31, 1015-1027 (1960).

2. Hoadley, A.B.: On the probability of large deviations of functions of several C.D.F.'s. Ann. Math. Stat. 38, 360-381 (1967).

3. Lewis, J.T., Pulè, J.: The equilibrium states of the free Boson gas. To appear.

4. Kac, M.: Private communication.

5. McKean, H.P., Singer, I. M.: Curvature and the eigenvalues of the Laplacion. J. Diff. Geom. 1, 43-69 (1967).

6. Feller, W.: An introduction to probability theory and its applications. Vol. 2. London: Wiley and Sons 1966.

7. Loeve, M.: Probability theory. 3rd edition. Princeton, New Jersey: Van Nostrand 1963.

8. Karlin, S.: Total positivity. Stanford Univ. Press (1968).

9. Ruelle, D.: Statistical mechanics. New York: W. A. Benjamin Inc. 1969.

10. Cannon,J.T.: Infinite volume limits of the canonical free Bose gas states on the Weyl algebra. To appear.

11. Huang, K.: Statistical mechanics. London: Wiley and Sons 1967.

E. B. Davies

Mathematical Institute

University of Oxford

England 